\title{
Towards Biomedical Problem Solving in a Game Environment
}

\author{
Yang Cai ${ }^{1}$, Ingo Snel ${ }^{2}$, B. Suman Bharathi ${ }^{2,3}$, Clementine Klein ${ }^{4}$, \\ and Judith Klein-Seetharaman ${ }^{1-3,5}$ \\ ${ }^{1}$ School of Computer Science, Carnegie Mellon University, Pittsburgh, PA15213, USA \\ ycai@andrew. cmu .edu \\ ${ }^{2}$ Institute for Organic Chemistry, University of Frankfurt, 60439 Frankfurt, Germany \\ ingosneleaol.com \\ ${ }^{3}$ Institute for Biological Information Processing, Research Institute Jülich, 52425 Jülich, \\ Germany \\ bsuman_1979@yahoo.com \\ ${ }^{4}$ Berufskolleg Kartäuserwall, Abteilung Medien, Kartäuserwall 30, 50678 Köln, Germany \\ clekle@hotmail.com \\ ${ }^{5}$ Department of Pharmacology, University of Pittsburgh Medical School, Pittsburgh, \\ PA15261, USA \\ judithks@cs.cmu.edu
}

\begin{abstract}
Biomedical systems involve complex interactions between diverse components. Problem solving in such systems requires insight, i.e. the capability to make non-obvious connections. In this paper, we present a game-based problem solving environment, where users can explore biological interactions with navigation on atomic to macroscopic scales, role-play, and networked collaboration. The study investigates the system architecture of the biological game, bio-morphing characters, and bio-interactions with biosensing and biodynamics. The prototype has been implemented on PC and tested in a preschool environment where users have little knowledge in biology. The experiment shows that the game greatly inspired users both in concept learning and entertainment.
\end{abstract}

\section{Introduction}

Rapid advances in the convergent technologies "nano-bio-info-cogno", referring to nanoscience and -technology, biotechnology and biomedicine, information technology and the cognitive sciences, are believed to have the potential to result in a "comprehensive understanding of the structure and behavior of matter from the nanoscale up to the most complex system" if these advances are exploited in a synergistic fashion [1]. In biomedicine, high-throughput methodology now allows the accumulation of unprecedented amounts of scientific data, such as genome sequences, gene expression profiles, structural and functional proteomic data. These advances have stirred great hopes for understanding and curing diseases, but the quantity of data requires convergence with information technology to interpret and utilize these data to advance human performance and quality of life [1]. This requires an understanding of 
the complex interactions between the components of biomedical systems by both domain and non-domain experts. This is particularly challenging in the biomedical domain because of the massive data and knowledge accumulation. Facilitating convergence of nano-bio-info-cogno technologies therefore requires a novel Problem Solving Environment (PSE) for the biomedical domain.

A biomedical PSE should be a computer system that provides all the computational facilities needed to immerse into a biomedical problem. It should hide the intricacies of computer modeling of physical phenomena so that the user can concentrate on developing an approach to cure a disease for example. The PSE has to be scientifically accurate and include access to the state-of-the-art in available data, knowledge and technology without requiring the user to bring domain expertise and extensive experience with the technical intricacies of the PSE that would present the user with tiresome activation barriers. Novel approaches are being developed, for example a storytelling system has been presented to fertilize multidisciplinary biomedical problem solving [2]. Furthermore, modern biomedical education makes extensive use of visualization of biomedical processes and concepts, for example the publication of the human genome sequence was accompanied by a CD-ROM that presented genome background as well as DNA sequencing techniques in animations [3]. However, these visualization tools are mostly designed to complement traditional teaching techniques and are not interactive. In contrast, interactive virtual laboratories have been developed for chemical and biomedical laboratory experiments (e.g. [4]), but these are targeted to students and researchers for solving very specific problems. Such systems provide too much domain specific information and too little insight for solving discovery problems for inexperienced users to serve as an integrated PSE.

Problem solving in biomedical systems requires insight, i.e. the capability to make non-obvious connections between the complex interactions of the components of these systems [5]. Such insightful solutions can often be found in an interactive and visual PSE, as demonstrated for example by the fact that despite the modern numerical computing technologies, biophysicists today still use Gedanken experiments for concept development [6]. Although there are many virtual reality three-dimensional molecular models available, biochemists still use hand-made models for intuitive reasoning. It is striking that simple intuitive simulation is still one of the most powerful approaches to creative problem solving.

Since the early days of artificial intelligence, issues of modeling scientific reasoning and its representation, in particular for those connected with everyday knowledge of the behavior of the physical world, have been studied [7]. At least two aspects have been explored: multiple representation and qualitative reasoning. Computation with Multiple Representations (CaMeRa) is a model that simulates human problem solving with multiple representations, including pictures and words [8]. CaMeRa combines a parallel network, used to process the low-level pictorial information, with rule-based processes in higher-level pictorial and verbal reasoning. Furthermore, many AI systems have been developed to simulate the cognition about physical and biological knowledge. What will happen if we spill a glass of milk on the floor? For humans, the answer is common sense, but understanding this process is non-trivial for computers. To arrive at an exact solution, the computer has to solve a set of non-linear partial 
differential equations of hydrodynamics that are computationally intractable even for simple boundary conditions [9]. A few studies have focused on the qualitative simulation of physical phenomena. Thus, Gardin uses two-dimensional diagrams to represent physical objects and their interaction [10] and Forbus uses the fuzzy language of "qualitative physics" to model the physical variables [11]. Lower-resolution qualitative models have made significant impact in many fields, including biology. A typical example is the Game of Life, a "Cellular Automaton" [12]. A cellular automaton is an array of identically programmed automata, or "cells", which interact with one another. The state of each cell changes from one generation to the next depending on the state of its immediate neighbors. By building appropriate rules, complex behavior can be simulated, ranging from the motion of fluids to outbreaks of starfish on a coral reef. Even if the line of cells starts with a random arrangement of states, the rules force patterns to emerge in life-like behavior. Empirical studies by Steven Wolfram [13] and others show that even the simple linear automata behave in ways reminiscent of complex biological systems. In light of this discovery, we intend to use simple biological characters to generate dynamic interactions.

Here we present a computer game as a novel environment for biological problem solving, where it provides a real-time interactive platform for users. The goal of this study is to develop a game-based PSE for users to explore multi-modal interactions inside a biological system. It includes essential biological simulation models for the immune system and the blood system. It allows users to manipulate and to participate in the interactions of the components of the system. The biological characters are simulated by software agents in the game.

\section{System Architecture}

As a test bed for the development of a game-based PSE for biomedical science, we designed a scientific problem that is derived from our ongoing research projects. By applying computational language technologies to the large amounts of whole genome sequence data publicly available, we have identified "genome signatures" that may provide new the development of vaccines against pathogenic microorganisms such as Neisseria [14]. The biomedical problem to be explored here is to find treatment for fatal meningitis. The new idea for the biomedical PSE is to develop an interactive interface modeled after traditional game engines, that teaches users without background in biology the understanding of this research problem ranging in hierarchy from atomic to macroscopic scales. In this hierarchy, the macroscopic level is that of infection of a human body with Neisseria. Fighting the infection at this level, however, requires a molecular level understanding of the processes involved. The goal is to provide the user with the necessary insight to creatively generate and test possible approaches to solving this problem using the PSE. The PSE contains three interaction modes: role-play, voyage and networked problem solving. Users can select a mode from the main menu. 
- Role-Play. The system allows the user to be a biological character in the game. Cognition Science shows that role-play is an important way to stimulate creative ideas. It enables the user to have an intimate connection to the character. Also, personalization of a biological character makes a game more interactive.

- Voyage. The user can navigate through the biological system in the game. This gives the user an opportunity to look at the interactive components from different aspects, for example, travelling through capillaries and tissues. The voyage allows exploration at the user's chosen leisure, accommodating users with various backgrounds.

- Distributed Problem Solving. The game engine allows users to play the game over the Internet so that users can solve large problems in a collaborative way. For example, some users can play macrophages and others can play bacteria. The distributed problem solving enables diverse game strategies and more excitement of the game.

BioSim version 1.0 is a rapid prototype for this PSE. It is a two-stage game that simulates the journey of red blood cells and white blood cells (macrophages). The goal is to introduce the basic concepts of cellular interaction and the human immune system. The game begins with an animated scene of a blood stream with red and white cells moving passively with the heartbeat. Using a mouse and the arrow keys, the player can take the role of a biological character, for example a macrophage, and navigate inside the blood stream. The user can also actively squeeze out in capillary regions to access tissue that is infected by bacterial cells, which multiply at a certain speed. Screen shots of these processes are shown in Fig. 1.
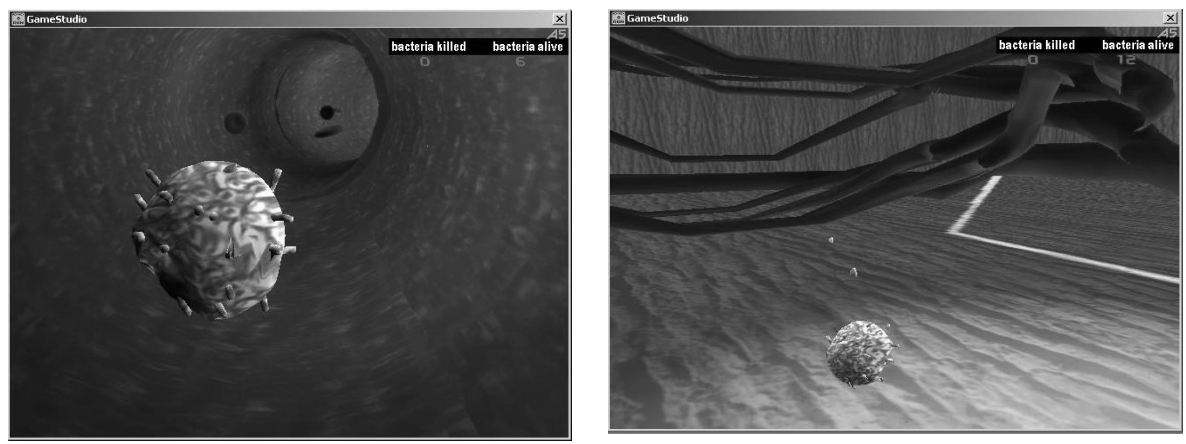

Fig. 1. The Macrophage (white blood cell) is inside the blood stream with red blood cells (left). After actively moving out of the blood stream, the macrophage approaches bacteria that infected human body tissue (right)

\section{Biological "World Model"}

In game design, "world models" are similar to the theatre stage or film scene with which actors and characters interact. A world model is often static and large in size. In this project, we developed a comprehensive world model that includes the vascular 
system with artery, veins and capillaries, as well as tissues (Fig. 2). In this world, the user can fly, walk or run through as one of the biological characters. In the prototype BioSim 1.0, we developed two scenes: inside the capillary and outside of the capillary. The transition of the scenes is possible by "squeezing" a character actively from the capillary to the tissue.
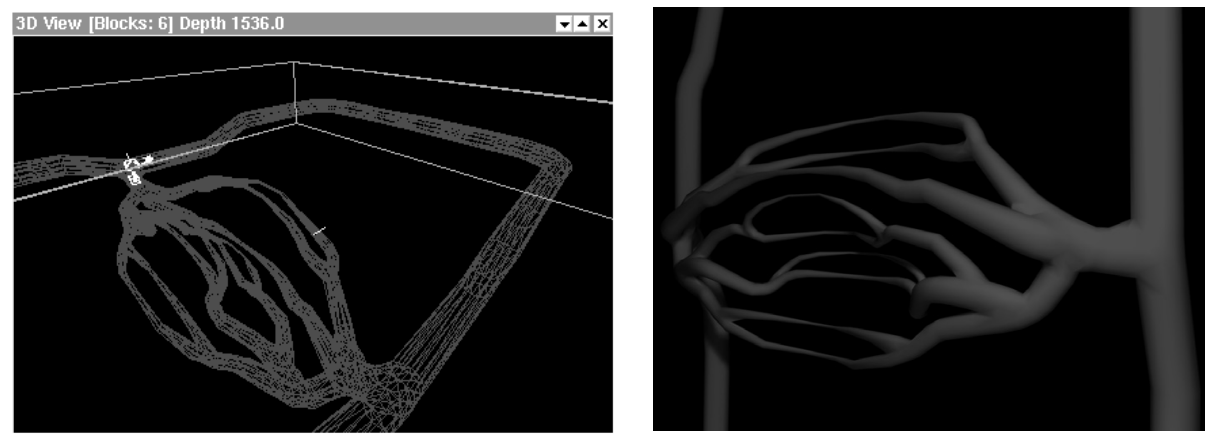

Fig. 2. Vascular system represented in the game. The 3-D wireframe model (left) and the 3-D photorealistic model (right) include arteries and capillaries

\section{Biological Characters}

So far, we have defined the following 3D animated characters that simulate biological behavior: bacteria, macrophages, and red blood cells. For the stand-alone characters, we apply bio-morphing to assign key frames to them. Bio-morphing is accomplished by digitizing deformed shapes from microscopic images of organisms, building wire frames and attaching texture and color skins. The transitions of each character are represented by a state machine (Fig. 3, left). For example a macrophage's states include the transitions to deform, shrink, eat, walk and die.

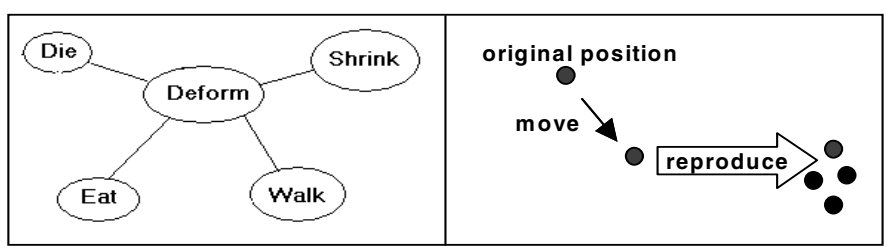

Fig. 3. State machine for a macrophage (left) and dynamics of an organism modeled by cellular automata (right)

\section{Biological Interactions}

Interaction is the key to computer games, and we believe similarly to an efficient biomedical PSE. We therefore allow the user to control the behavior of biological characters by realistic and scientifically accurate bio-interactions. These consist of bio-sensing and bio-dynamics. 
Bio-sensing describes the ability of biological systems to sense environmental stimuli. For example, white blood cells can "smell" bacteria and move toward them by chemotaxis. To simulate such sensing capabilities, we define a circular envelope around the character as a sensing region. When the target is inside the region, the character will move towards the target and engage in interactions with it.

Bio-dynamics simulate biological processes realistically. This is one of the primary components adding excitement to the game. For each character, we define its interactive modes, such as motion, reproduction and death. Taking bacteria for example, we use the following rules (Fig. 3, right):

- Autonomous motion. Given a fixed duration, each bacteria moves a distance $\mathrm{x}$ at angle $\mathrm{y}$, where $\mathrm{x}$ and $\mathrm{y}$ are random values. The distance should not exceed the maximal distance.

- Reproduction. Given a predefined duration, each bacterium reproduces a copy of its own which is placed beside its original position. The Logistic growth model [15] adequately describes the reproduction process of simple organisms over limited time periods by equation (1), where $M$ is the carrying capacity of the population.

$$
B_{n+1}=B_{n}+r B_{n}\left(1-B_{n} / M\right) \text {. }
$$

- Death. If a bacterium's life cycle is over or if other cells eat it, it is removed from the scene.

\section{Implementation}

The prototype of BioSim 1.0 is implemented on PC. Photorealistic 3D models of components of the system were created with 3D Studio Max and imported into Game Studio 3D. 3D Modeler of GameStudio 3D was used to create the game scenes, biomorphed characters and the integration of the world/character dynamics and interactions. C-script, a C-style language, was used to encode the bio-dynamics and biosensing behaviors. Game Studio is run under the Windows operation system. It provides capability for either single user or multiple users across the Internet.

\section{A Case Study}

We conducted experiments in the effectiveness of the game to raise an awareness of the important issues in biomedical research on users with no background. The ideal group at this stage of implementation of the game is young children, for two reasons. One, children are unbiased and without background. Second, children learn optimally when the material to be learned is presented to them in an accurate way to avoid the build-up of incorrect models by implicit learning [16]. Implicit learning of correct biomedical concepts by children therefore requires the same fundamental issue of scientific accuracy as other users will require once the game reaches the stage of 
providing a PSE for users with any background. We tested BioSim 1.0 on 14 children at KinderCare, Cranberry, PA, on August 9th, 2002 and February 25, 2003. We let four- and five-year-old children play with the game on a laptop and focused our attention on strategic aspects and active questioning in the children's behavior.

Two strategies were quantified, the speed of macrophage movement towards the bacteria (Fig. 4, left) and the use of antibiotics in aiding the killing of the bacteria (Fig. 4, right). All children learnt quickly to shift from fast pace chasing to slow pace chasing so that their capture rate was improved. We then tested a more challenging concept, that of usage of antibiotics to aid the killing of the bacteria. We included the ability of bacteria to develop resistance in our growth model. Thus, the children had to discover that antibiotics at some stage in the game no longer inhibit bacterial growth. This was only observed by a single 5-year old, all other children kept on administering antibiotics despite energy consumption and lack of effect (Fig. 4, right). These types of quantitative assessment of strategic behavior of users open novel ways to analyze learning of problem solving skills that would not be possible with conventional teaching methods.

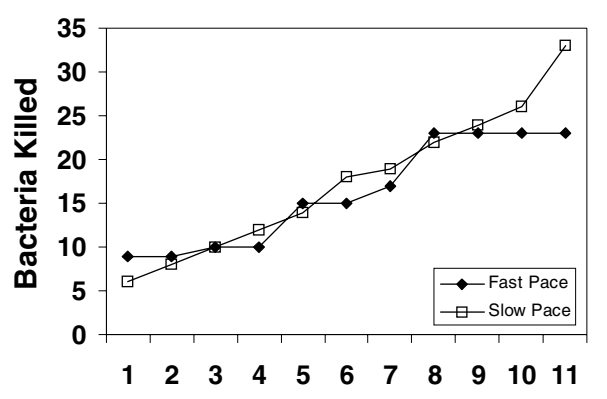

Time $\times 10$ [seconds]

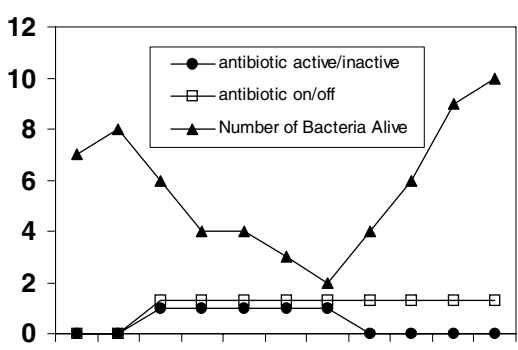

$\begin{array}{llllllllllll}1 & 2 & 3 & 4 & 5 & 6 & 7 & 8 & 9 & 10 & 11\end{array}$

Time x 10 [seconds]

Fig. 4. Strategies against bacterial infection that can be explored in the game, speed of macrophage movement (left) and use of antibiotics (right). The fast pace strategy often leads to missing targets. The slow pace strategy gains steady capture rate. The use of antibiotics can be limited by gradual development of bacterial resistance. At that point, administration of drug does not inhibit bacterial growth.

Next, we asked learning assessment questions, such as "How does the macrophage get out of the capillary?" or "How do you kill bacteria?" The children used intuitive metaphors, for example the analogy of "vacuum" and "crash into" to describe how the macrophage attacks bacteria. This shows that the players are very sensitive to the intimate design details of the game, which opens a window for game developers to encode very subtle knowledge about complex biological interactions.

Finally, we tested the game-induced stimulation of questioning in the children. The results are summarized in Table 1. The five-year-old children asked several meaningful questions, for example: "Are bacteria germs?" "Where do the white cells go?" "What's a red cell?" "Where do the bacteria live?" "Is the macrophage good or bad?" Overall, four-year-old children asked fewer questions, and most of their questions were not relevant, for example, "Do you have other games?" "I don't want my 
head eaten off." These observations suggest that there may be a turning point between ages 4 and 5 where a PSE can become effective.

Table 1. Comparison of the reactions of two groups of children to the game. The children in the first group were 4 years old, those in the other were 5 years old. Each group consisted of 7 children, and the total number of children tested was 14 .

\begin{tabular}{lll}
\hline Observation & 4-year old & 5-year old \\
\hline Asked relevant questions & 0 & 4 \\
Controlled the game successfully & 2 & 5 \\
Described bacterial growth behavior & 1 & 5 \\
Described macrophage behavior & 2 & 6 \\
\hline
\end{tabular}

\section{Further Developments in Game Design}

We are currently in the process of adding more capabilities for the user to interact with different components of the game, and also with multiple players connected to the same game via the Internet (Distributed Problem Solving). In the next version of the game, the user will choose between two aims, rather than playing the role of a single biological component. This modification will allow the user to assume the roles of multiple biological characters, thus studying their individual influence on a particular aim. These aims will be either to induce an infection with Neisseria and ensure its successful propagation in the human body or to fight the Neisseria infection. To enable more complex means of interaction with the biological world, each user will be equipped with a ship that allows for effective immersion into the environment (Fig. 5, upper left). The ship provides a means of transportation (Voyage) and action (Role Playing). Each activity is determined by availability of "energy points", which have to be carefully balanced to minimize consumption and maximize effectiveness. The user knows the status of energy points via a control panel, which also provides for the various possibilities of action (Fig. 5, upper right). For example, in a state of high energy, the user can afford to travel actively with the ship to a point of infection. However, in a state of low energy, the user would choose to travel passively with the blood stream. This will allow the user to further develop decision-making skills in a biological PSE.

There will also be additional biosensing capabilities available through the control panel, for example a histamine sensor (Fig. 5 lower left) and mechanisms of the immune system to distinguish self from non-self (Fig. 5, lower right). This will allow introduction of molecular level information, for example the user will need to use molecular docking of the immune system's antibody structures to those of the bacterial surface structures. This will train users to view protein structures and understand the mechanisms of complementarities of two structures. The player seeking to evade the immune system would need to develop strategies to evade antibody marking, e.g. through surface mutation. Thinking about possible strategies from each point of view will allow the user to gain deep insight into the factors controlling the health of the 
organism, from the molecular to the macroscopic level, ultimately aiding in the development of novel solutions for biomedical problems such as the Neisseria infection.
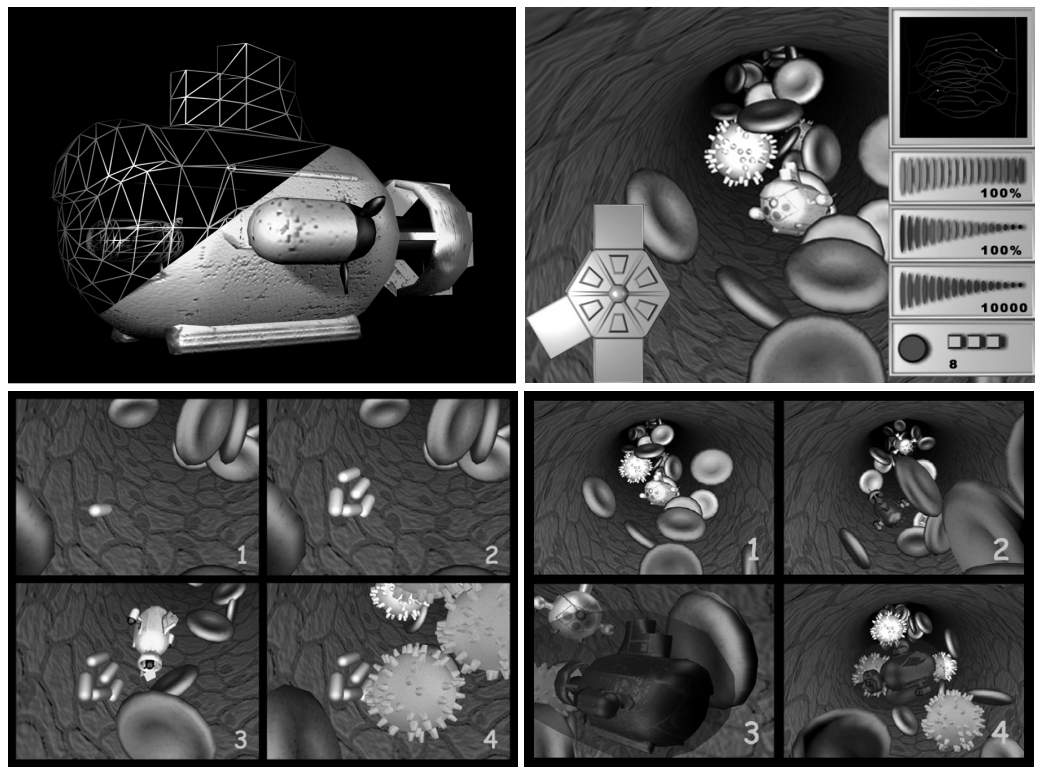

Fig. 5. Immersion of the user in the PSE. A ship provides transportation through the body (upper left). A control panel inside the ship has sensing and action capabilities (upper right). Marking of bacteria with histamines. Bacteria that have not been marked can divide undisturbed (gray bacteria in 1,2). When the ship (yellow) approaches the bacteria (3), the histamine sensor identifies the bacterial infection and the user can mark them (color change). After marking, the user can attract macrophages that will "eat" the bacteria (4) (Lower left). Increased complexity in future game. Internet connectivity will allow multiple users to participate in collaboration or as antagonists in a single game (yellow ship, 1, and blue ship, 2). Since ships are not-self, the human immune system would identify them as such. Therefore, one ship can mark another ship using immune system tools, i.e. antibodies. This is represented by the halo of the blue ship. Once labeled with antibodies, the user can again direct macrophages to "eat" the blue ship (lower right).

\section{Summary}

Future biomedical problem solving is beyond traditional means because of the existing challenges in cross-disciplinary communication and interpretation and utilization of vast quantities of available biomedical data. We want to build a virtual PSE that combines advanced computer graphics, computer vision, artificial intelligence technologies and creative instruction technologies. In this PSE, cross-disciplinary education will be on-demand, entertaining and interactive. This will allow focus on discovery and creativity rather than one-way tutoring. Towards this long-term goal, here, we have presented a game-based PSE, where users can explore complex biological inter- 
actions with navigation, role-play, and networked collaboration. The study investigates the system architecture of the biological game, bio-morphing characters, and bio-interactions with bio-sensing and bio-dynamics. The game is based on realistic biological models, such as logistic growth models of simple organism reproduction and immigration models of cell movements. The prototype has been implemented on PC and tested in a preschool environment where users have little knowledge in biology. The experiment shows that the game greatly inspired users both in concept learning and entertainment suggesting that the game-based PSE helps users to learn biosystem dynamics and multiple object interactions.

Acknowledgements. This work was supported by the Alexander von HumboldtFoundation and Zukunftsinvestitionsprogramm der Bundesregierung, Germany.

\section{References}

1. Roco, M.C. and W.S. Bainbridge: Overview. In: Converging Technologies for Improving Human Performance: Nanotechnology, Biotechnology, Information Technology, and Cognitive Science, Bainbridge, W.S. (ed.) National Science Foundation: Arlington, Virginia. (2002) 1-23

2. Kuchinsky, A., K. Graham, D. Moh, A. Adler, K. Babaria, and M.L. Creech: Biological storytelling: A software tool for biological information organization based upon narrative structure. AVI (2002)

3. http://www.sciencemag.org

4. http://ir.chem.cmu.edu/irproject/applets/virtuallab/

5. Cai, Y.: Pictorial Thinking, in Journal of Cognition Science.: Taiyuan, China (1986)

6. Hayden, T.: The inner Einstein, in US News (2002)

7. McCarthy, J. and P.J. Hayes: Some philosophical problems from the standpoint of artificial intelligence, in Machine Intelligence, Michie, D. (ed.) Edinburgh University Press: Edinburgh (1969)

8. Simon, H.: Models of Thoughts. Vol. II. Yale Press (1989)

9. Feynman, R.: The Feynman Lectures on Physics. Reading, Massachusetts: AddisonWesley (1963)

10. Gardin, F. and B. Meltzer: Analogical representations of naive physics. Artificial Intelligence 38 (1989) 139-159

11. Forbus, K.D.: Qualitative Process Theory. Artificial Intelligence 24 (1994) 85-168

12. Gardner, M.: Mathematical games: The fantastic combinations of John Conway's new solitaire game "life". Scientific American 223 (1970) 120-123

13. Wolfram, S.: A new kind of science Wolfram Publishing (2002)

14. Ganapathiraju, M., D. Weisser, R. Rosenfeld, J. Carbonell, R. Reddy, and J. KleinSeetharaman: Comparative n-gram analysis of whole-genome protein sequences. in Proc. Human Language Technologies San Diego (2002)

15. Guyton, A.C. and Hall, J.E.: Textbook of Medical Physiology Philadelphia: W.B. Saunders Co (1997)

16. Perrig, P. and W.J. Perrig: Implicit and explicit memory in mentally retarded, learning disabled, and normal children. Swiss J of Psychology 54(2) (1995) 77-86 